\title{
Influencia del programa de recuperación de espacios libres para fomentar cultura ambiental en estudiantes de una institución educativa peruana, 2020
}

\author{
Visitación Tello Sallo de Cabrera \\ visitacióntello901@gmail.com \\ https://orcid.org/0000-0001-6701-064X \\ Universidad San Ignacio Loyola -Lima, Perú \\ Jaime David Menacho Carhuamaca \\ menachohunter1979@gmail.com \\ https://orcid.org/0000-0002-2496-4280 \\ Universidad César Vallejo-Lima, Perú \\ Rocío del Pilar Cairampoma Medrano \\ cairampoma_24_12@hotmail.com \\ https://orcid.org/0000-0003-1990-1338 \\ Universidad San Ignacio Loyola -Lima, Perú
}

\section{RESUMEN}

La presente investigación se realizó con el objetivo de determinar la influencia de un programa educativo entorno a la recuperación de los espacios libres para fomentar la cultura ambiental en estudiantes de educación básica regular. La metodología se fundamentó en un diseño experimental y se contó con una población estudiantil de 450 participantes del nivel secundaria. Asimismo, se utilizó la encuesta y el cuestionario como instrumento de recojo de información. Se midió cuatro dimensiones de la variable cultura ambiental. Los resultados evidenciaron que los factores motivacionales y participativos de los estudiantes al experimentar la vivencialidad de las acciones ejecutadas, han influido en el desarrollo pertinente de programas educativos. Se concluyó, que las actividades efectuadas en el programa planificado han permitido el fortalecimiento del conocimiento ambiental, la cultura preventiva y el cuidado de los espacios libres en estudiantes de educación básica regular

Palabras clave: cultura ambiental; espacios libres; estrategias educativas; programas educativos; biodiversidad 


\title{
Influence of the program of recovery of free spaces to promote environmental culture in students of a peruvian educational institution, 2020
}

\begin{abstract}
The present researching was carried out with the objective of determining the influence of an educational program around the recovery of free spaces to promote environmental culture in students of regular basic education. The methodology was based on an experimental design and there was a student population of 450 participants from the secondary level. Likewise, the survey and questionnaire were used as an instrument for collecting information. Four dimensions of the environmental culture variable were measured. The results showed that the motivational and participatory factors of the students when experiencing the experiences of the actions carried out, have influenced the pertinent development of educational programs. It was concluded that the activities carried out in the planned program have allowed the strengthening of environmental knowledge, the preventive culture and the care of free spaces in students of regular basic education
\end{abstract}

Keywords: enviromental culture; free spaces; educational strategies; educational programs; biodiversity

Artículo recibido: 15 noviembre. 2021 Aceptado para publicación: 10 diciembre 2021 Correspondencia: visitacióntello901@gmail.com

Conflictos de Interés: Ninguna que declarar 


\section{INTRODUCCIÓN}

En los últimos años, la educación como proceso formativo y las instituciones educativas vienen desarrollando de manera conjunta una labor fundamental en la conservación de los espacios públicos, la biodiversidad existente y los ecosistemas contando con la participación de la comunidad educativa en la solución de problemas relacionados con el entorno proporcionándole conocimiento, actitudes, competencias para poder interpretar la realidad problemática (Simoes, Yanes y Álvarez, 2019). En ese sentido, la predisposición de una pertinente enseñanza ambiental mediante una cultura preventiva y de conservación brindará a los estudiantes las habilidades indispensables para adoptar medidas adecuadas y tomar decisiones convenientes para el medio en el que se habita y se desenvuelve a diario.

Del mismo modo, en el camino de las posibilidades de la formación de los estudiantes es necesario tener presente la innovación. Dentro de esta premisa se pretende buscar un papel más activo del estudiante en el proceso de un aprendizaje vivencial, a su vez es necesario obligar al abandono de métodos tradicionales de instrucción a favor de las estrategias participativas y en contacto con el entorno, unido a éste la búsqueda de la influencia de la escuela en la comunidad para su integración a favor de contribuir más eficazmente en la labor educativa (Menacho y Cadenillas, 2021; Farroñan, 2017)

El problema a desarrollar se ha formulado en ¿Cómo influye los programas de recuperación de espacios libres para fomentar la cultura ambiental de los estudiantes de una institución educativa peruana? Se ha direccionado en determinar la posibilidad de romper con las metodologías tradicionales para la formación de los estudiantes buscando la participación consciente de ellos en la tarea de identificar, revalorar y conservar el patrimonio natural y cultural como parte del espíritu y para ello tendrá que conocerlo y asumirlo. Así mismo, desde la perspectiva de Severiche, Gómez y Jaimes (2016) se busca la participación en la identidad, cuidado y protección del medio ambiente y de las áreas verdes y sus dimensiones planeación y organización de áreas verdes, dirección y ejecución de áreas verdes, sostenibilidad de áreas verdes, tomando en cuenta la actitud, comportamiento y conocimiento de los estudiantes, buscando desarrollar su cualidad artística acorde a las áreas y proyectos que se presentan. En la actualidad experiencias europeas, manifiestan que las extensas poblaciones han hecho que aumenten en forma descontrolada la expansión de las urbes, y sus consecuencias de sostenibilidad es negativa 
y proponen el perfil de anillos verdes, describen su contexto y el enfoque de planificación (Aguado, Barrutia, Echebarria, 2017; Maciel y Barboza, 2015). También se ha descrito en el estudio experiencias realizadas en otros lugares del contexto latinoamericano con la finalidad de mejorar el marco teórico de la cultura ambiental

Tratar el tema de educación ambiental es concientizar a la población sobre la necesidad de preservar el ambiente para una calidad de vida en las generaciones presentes y futuras, según la variable cultura ambiental la transcendencia de la educación ambiental como es de optimizar la cultura presente y futura y analiza de manera cualitativa la literatura científica disponible en base de datos como Science Direct, Scielo, Redalyc y páginas web oficiales (Checa, 2016). Por ello, la educación ambiental entonces es fundamental para la generación en el cambio de actitud y aptitud para lograr el equilibrio entre el ser humano y su entorno

El diseño de áreas verdes desde la perspectiva ecológica, pone énfasis en los espacios abiertos, que pueden ser ponentes de la biodiversidad en el espacio urbano, la investigación trata en la búsqueda de alternativas del uso extensivo del césped, proponiendo soluciones estratégicas, atendiendo la sostenibilidad en espacios de intervención; muestra de ello presentan una experiencia desarrollada durante un año en relación a la evolución de unas praderas en dos zonas verdes en la localidad española de Illescas, perteneciente al Municipio de Toledo, que han podido reducir costos e incremento de biodiversidad en concordancia con alternativas de mejora sostenible (Equipo de la Federación Española de Municipios y Provincias, 2016)

A nivel de países latinoamericanos vienen progresando la presencia macroeconomía social y por ende surgen problemas de larga data de no solución, como es la concentración de macrocefalia urbana, por falta de eficiencia y transparencia de los recursos naturales, la investigación, de la calidad de vida con relación a la situación propia de Argentina, en el que Abasto (2020) propone cuatro ejes a tomar en cuenta el desarrollo sostenible, la movilidad verde, la red ferroviaria, el manejo sostenible de los recursos, la eficiencia energética para superar el extractivismo en el contexto de CONASUR. Por otro lado, la Universidad Católica de Chile realizó para la sostenibilidad de las áreas verdes un estudio sobre "estimación de costos de mantención de áreas verdes urbanas" que tiene que ver con la tipología de las áreas urbanas según sus formas y funciones para representar la diversidad, estimando rangos de costos de mantención para cada tipología, recomendando 
diseño y operación para disminuir el gasto, tomando muestras habitantes por área verdes (Cabrera, 2021). En la zona de Yucatán-México se realizó una experiencia sobre áreas verdes y el arbolado una de las dimensiones ecológicas social y económica de la sostenibilidad urbana en la zona de Mérida, su metodología se basó en el uso de satélite para captar la cobertura arbórea y uso Google Earth como herramienta de medición y cálculo de superficies recorridos de campo, llegando a la conclusión según resultados que las áreas verdes y cobertura arbórea se asocia al mercado de vivienda y sus tipologías, a la sucesión de gestión, gobernanza, y participación social (Moreno Fernández y Pérez García, 2018).

En Colombia se efectuó una investigación científica sobre la cultura ambiental, un estudio desde las dimensiones de valor, creencias, actitudes y comportamientos ambientales, según Miranda (2013) refiere que la cultura ambiental es la manera como los seres humanos se vincula con el medio ambiente y para entenderla se debe comenzar por el estudio de los valores, a su vez establecen las creencias y las actitudes, que son elementos que dan sentido al comportamiento ambiental, enfocándose de acuerdo a las características propias de la comunidad. En ese sentido, la cultura es considerada un patrimonio general, un componente esencial del medio ambiente que involucra no solo los espacios públicos, sino la conservación, y concientización ciudadana para la consolidación de una cultura ambiental favorable (García y Guerrero, 2006; Sánchez Aizcorbe, 2017). En el contexto ecuatoriano, el estudio de Núñez Aldaz, Hayk y Bejas Monzant (2021) se predispuso entorno a los principios educativos de la educación y cultura ambiental como eje clave para la modernización de la enseñanza y el diseño curricular interdisciplinario. Así, se propuso afianzar la necesidad de ecologizar las relaciones sociales, fomentar la cultura preventiva del cuidado de los espacios públicos con una visión de desarrollo sostenible

A nivel nacional en la Bahía de Paracas después de muchos estudios lograron investigar como las especies marinas de Paracas no se le estaba dando el tratamiento debido en ellas especies marinas como lobo de mar, pingüinos, foca de mar estaban siendo abandonados, estos trabajos arduos lograron a realizar las eco playas, brindando atención, respetando los espacios de este hábitat (Guezel y Wickel, 2015), la costa verde de Lima y las áreas verdes tienen un potencial de integración, el estudio de espacios naturales busca conectar una red de parques que mejore la conectividad en el conjunto urbano, además el medio 
abiótico que lo sostiene, mejora las condiciones naturales en la ciudad y bienestar de la población y su uso recreativo (Peña, 2015; Vila, 2017)

A nivel local, en los distritos capitalinos de Santiago de Surco al sur, Chaclacayo al este y Comas al norte, en cuanto al uso de los espacios públicos según la normatividad de Lima Metropolitana y la gestión municipal, seleccionaron parques representativos de los diversos niveles económicos en torno a la percepción de calidad y relevancia de los habitantes de los distritos mencionados (Quispe, 2017; Capristan, 2017; Rojas, 2016) dichos parques a pesar de contar con superficies de gran consideración donde se llevan numerosas actividades, estos no están bajo un sistema de gestión legal apropiado para asegurar su mantenimiento y mejora para el futuro, y menos aún para contribuir a la calidad de vida de los vecinos. Los hallazgos de estos investigadores han permitido mejoras sustantivas con el estado actual de los parques estudiados como espacios públicos

\section{MÉTODO Y MATERIALES}

La investigación fue de tipo aplicada, enfoque cuantitativo, diseño experimental se aplicó a un grupo de participantes que es medido en la observación 1 y observación 2, nivel pre experimental, la población es de 450 estudiantes y la muestra se conformó de 50 estudiantes, la técnica utilizada fue la encuesta y el instrumento es un cuestionario, las dimensiones que fueron medidas fueron: actitudes positivas, intenciones del comportamiento, intenciones del conocimiento ambiental, intenciones de la cultura ambiental. Es preciso acotar que el estudio fue desarrollado a través de un programa en 30 sesiones de aprendizaje en el que se aplicó las siguientes temáticas: 1. Sensibilización sobre medio ambiente, 2. Cultura ambiental, 3. Dimensiones de las áreas verdes, 4.Caracteristicas de los seres vivos, 5. Composición química de los seres vivos, 6. Niveles de organización de los seres vivos, 7. Clasificación de los seres vivos, 8.Características de las plantas, 9. Clasificación de las plantas, 10. Plantas briofitas y pteridofitas, 11.Plantas gimnospermas y angiospermas, 12. La nutrición de las plantas, 13 la relación en las plantas, 14. Adaptaciones de las plantas, 15 la reproducción en las plantas, 16. La flora en el Perú, 17. El ecosistema, 18. Organización del ecosistema, 19. Estudio de las poblaciones, 20. Las relaciones bióticas, 21. Evolución de los ecosistema, 22. ¿Qué es la desforestación?, 23. ¿A qué se llama contaminación? 24. ¿Qué son el efecto invernadero y el calentamiento global?, 25. Biohuertos, 26. Hidroponía, 27. Jardines y reforestación, 28. Elaboración de afiches, carteles, mensajes y otros recursos que ayuden a publicar el 
cuidado del medio ambiente, 29. Reciclaje con las 3 R, 30. Elaboración de proyectos de plásticos y de neumáticos.

\section{RESULTADOS}

El procesamiento de la información en el programa informático SPSS ha permitido la obtención de los resultados del presente estudio que a continuación se podrá visualizar en las siguientes tablas:

Tabla 1. Prueba de Wilcoxon para actitudes positivas

\begin{tabular}{|c|c|c|c|c|}
\hline & & \multicolumn{3}{|c|}{$\begin{array}{c}\text { Actitudes positivas_postest - Actitudes } \\
\text { positivas_pretest }\end{array}$} \\
\hline \multicolumn{2}{|c|}{$\mathrm{Z}$} & \multicolumn{3}{|c|}{$-4,346^{\mathrm{b}}$} \\
\hline \multicolumn{2}{|c|}{ Sig. asintótica (bilateral) } & \multicolumn{3}{|c|}{, 000} \\
\hline \multicolumn{5}{|c|}{ a. Prueba de Wilcoxon de los rangos con signo } \\
\hline \multicolumn{5}{|c|}{ b. Se basa en rangos negativos. } \\
\hline \multicolumn{3}{|c|}{ Descriptivos } & Estadístico & Error estándar \\
\hline \multirow{13}{*}{$\begin{array}{c}\text { Actitudes } \\
\text { positivas_pretest }\end{array}$} & \multicolumn{2}{|l|}{ Media } & 10,92 & 281 \\
\hline & \multirow{2}{*}{\multicolumn{2}{|c|}{$\begin{array}{l}95 \% \text { de intervalo de Límite inferior } \\
\text { confianza para la media Límite superior }\end{array}$}} & 10,35 & \\
\hline & & & 11,49 & \\
\hline & \multicolumn{2}{|c|}{ Media recortada al 5\% } & 10,88 & \\
\hline & \multicolumn{2}{|l|}{ Mediana } & 11,00 & \\
\hline & \multicolumn{2}{|l|}{ Varianza } & 3,953 & \\
\hline & \multicolumn{2}{|l|}{ Desviación estándar } & 1,988 & \\
\hline & \multicolumn{2}{|l|}{ Mínimo } & 7 & \\
\hline & \multicolumn{2}{|l|}{ Máximo } & 15 & \\
\hline & \multicolumn{2}{|l|}{ Rango } & 8 & \\
\hline & \multicolumn{2}{|l|}{ Rango intercuartil } & 3 & \\
\hline & \multicolumn{2}{|l|}{ Asimetría } &, 537 & ,337 \\
\hline & \multicolumn{2}{|l|}{ Curtosis } &,- 272 & 662 \\
\hline \multirow{13}{*}{$\begin{array}{l}\text { Actitudes } \\
\text { positivas_postest }\end{array}$} & \multicolumn{2}{|l|}{ Media } & 11,72 & ,267 \\
\hline & \multirow{2}{*}{$\begin{array}{l}95 \% \text { de intervalo de } \\
\text { confianza para la } \\
\text { media }\end{array}$} & Límite inferior & 11,18 & \\
\hline & & Límite superior & 12,26 & \\
\hline & \multicolumn{2}{|l|}{ Media recortada al $5 \%$} & 11,77 & \\
\hline & \multicolumn{2}{|l|}{ Mediana } & 12,00 & \\
\hline & \multicolumn{2}{|l|}{ Varianza } & 3,553 & \\
\hline & \multicolumn{2}{|l|}{ Desviación estándar } & 1,885 & \\
\hline & \multicolumn{2}{|l|}{ Mínimo } & 7 & \\
\hline & \multicolumn{2}{|l|}{ Maximo } & 15 & \\
\hline & \multicolumn{2}{|l|}{ Rango } & 8 & \\
\hline & Rango intercuartil & & 2 & \\
\hline & Asimetría & &,- 320 &, 337 \\
\hline & Curtosis & &,- 044 & 662 \\
\hline
\end{tabular}


En cuanto al procesamiento de la dimensión actitudes positivas se obtuvo tanto a nivel pretest y postest un 10.92 y 11.72 respectivamente en un rango especifico entre 3 y 15 , lo que ha evidenciado una mejora significativa de 0.80 , así como la influencia del programa desarrollado en los estudiantes de una escuela básica regular.

\section{Tabla 2}

Prueba de Wilcoxon para intenciones del comportamiento

\begin{tabular}{lc}
\hline & $\begin{array}{c}\text { Intenciones de comportamiento_postest - Intenciones } \\
\text { de comportamiento_pretest }\end{array}$ \\
\hline $\mathrm{Z}$ & $-5,709^{\mathrm{b}}$ \\
Sig. asintótica (bilateral) &, 000 \\
\hline
\end{tabular}

a. Prueba de Wilcoxon de los rangos con signo

b. Se basa en rangos negativos.

\begin{tabular}{|c|c|c|c|}
\hline & Descriptivos & Estadístico & $\begin{array}{c}\text { Error } \\
\text { estándar }\end{array}$ \\
\hline \multirow{13}{*}{$\begin{array}{c}\text { Actitudes } \\
\text { positivas_pretest }\end{array}$} & Media & 9,82 &, 175 \\
\hline & $95 \%$ de intervalo de Límite inferior & 9,47 & \\
\hline & confianza para la media Límite superior & 10,17 & \\
\hline & Media recortada al $5 \%$ & 9,84 & \\
\hline & Mediana & 10,00 & \\
\hline & Varianza & 1,538 & \\
\hline & Desviación estándar & 1,240 & \\
\hline & Mínimo & 7 & \\
\hline & Máximo & 12 & \\
\hline & Rango & 5 & \\
\hline & Rango intercuartil & 1 & \\
\hline & Asimetría &,- 245 & ,337 \\
\hline & Curtosis &,- 050 & ,662 \\
\hline \multirow{13}{*}{$\begin{array}{c}\text { Actitudes } \\
\text { positivas_postest }\end{array}$} & Media & 11,74 & ,208 \\
\hline & 95\% de intervalo de Límite inferior & 11,32 & \\
\hline & confianza para la media Límite superior & 12,16 & \\
\hline & Media recortada al $5 \%$ & 11,73 & \\
\hline & Mediana & 12,00 & \\
\hline & Varianza & 2,156 & \\
\hline & Desviación estándar & 1,468 & \\
\hline & Mínimo & 8 & \\
\hline & Máximo & 15 & \\
\hline & Rango & 7 & \\
\hline & Rango intercuartil & 1 & \\
\hline & Asimetría &, 150 & ,337 \\
\hline & Curtosis & ,943 & ,662 \\
\hline
\end{tabular}


Además, en la dimensión intenciones del comportamiento se obtuvo tanto a nivel pretest y postest un 9.82 y 11.74 respectivamente en un rango especifico entre 3 y 15 , lo que ha evidenciado una mejora significativa de 1.92, así como la influencia del programa desarrollado en los estudiantes en dicha dimensión de una escuela básica regular.

\section{Tabla 3}

Prueba de Wilcoxon para intenciones del conocimiento ambiental

\begin{tabular}{|c|c|c|c|}
\hline & & \multicolumn{2}{|c|}{$\begin{array}{l}\text { Conocimiento ambiental_postest } \\
\text { Conocimiento ambiental_pretest }\end{array}$} \\
\hline \multicolumn{2}{|l|}{$\mathrm{Z}$} & \multicolumn{2}{|c|}{$-5,996^{\mathrm{b}}$} \\
\hline \multicolumn{2}{|c|}{ Sig. asintótica (bilateral) } & \multicolumn{2}{|l|}{, 000} \\
\hline \multicolumn{4}{|c|}{ a. Prueba de Wilcoxon de los rangos con signo } \\
\hline \multicolumn{4}{|c|}{ b. Se basa en rangos negativos. } \\
\hline \multicolumn{2}{|r|}{ Descriptivos } & Estadístico & $\begin{array}{c}\text { Error } \\
\text { estándar }\end{array}$ \\
\hline \multirow{13}{*}{$\begin{array}{c}\text { Actitudes } \\
\text { positivas_pretest }\end{array}$} & Media & 10,54 & ,254 \\
\hline & \multirow{2}{*}{$\begin{array}{l}95 \% \text { de intervalo de Límite inferior } \\
\text { confianza para la media Límite superior }\end{array}$} & 10,03 & \\
\hline & & 11,05 & \\
\hline & Media recortada al $5 \%$ & 10,54 & \\
\hline & Mediana & 11,00 & \\
\hline & Varianza & 3,233 & \\
\hline & Desviación estándar & 1,798 & \\
\hline & Mínimo & 6 & \\
\hline & Máximo & 15 & \\
\hline & Rango & 9 & \\
\hline & Rango intercuartil & 2 & \\
\hline & Asimetría &,- 057 & ,337 \\
\hline & Curtosis & ,270 & ,662 \\
\hline & Media & 13,50 & ,192 \\
\hline & \multirow{2}{*}{$\begin{array}{l}95 \% \text { de intervalo de Límite inferior } \\
\text { confianza para la media Límite superior }\end{array}$} & 13,11 & \\
\hline & & 13,89 & \\
\hline & Media recortada al $5 \%$ & 13,52 & \\
\hline & Mediana & 13,50 & \\
\hline & Varianza & 1,847 & \\
\hline Actitudes & Desviación estándar & 1,359 & \\
\hline & Mínimo & 11 & \\
\hline & Máximo & 15 & \\
\hline & Rango & 4 & \\
\hline & Rango intercuartil & 3 & \\
\hline & Asimetría &,- 076 & ,337 \\
\hline & Curtosis & $-1,691$ & ,662 \\
\hline
\end{tabular}


Asimismo, en la dimensión intenciones del conocimiento ambiental se obtuvo tanto a nivel pretest y postest un 10.54 y 13.5 respectivamente en un rango especifico entre $3 \mathrm{y}$ 15, lo que ha evidenciado una mejora significativa de 2.96, así como la influencia del programa desarrollado en los estudiantes en dicha dimensión de una escuela básica regular.

\section{Tabla 4}

Prueba de Wilcoxon para intenciones de la cultura ambiental

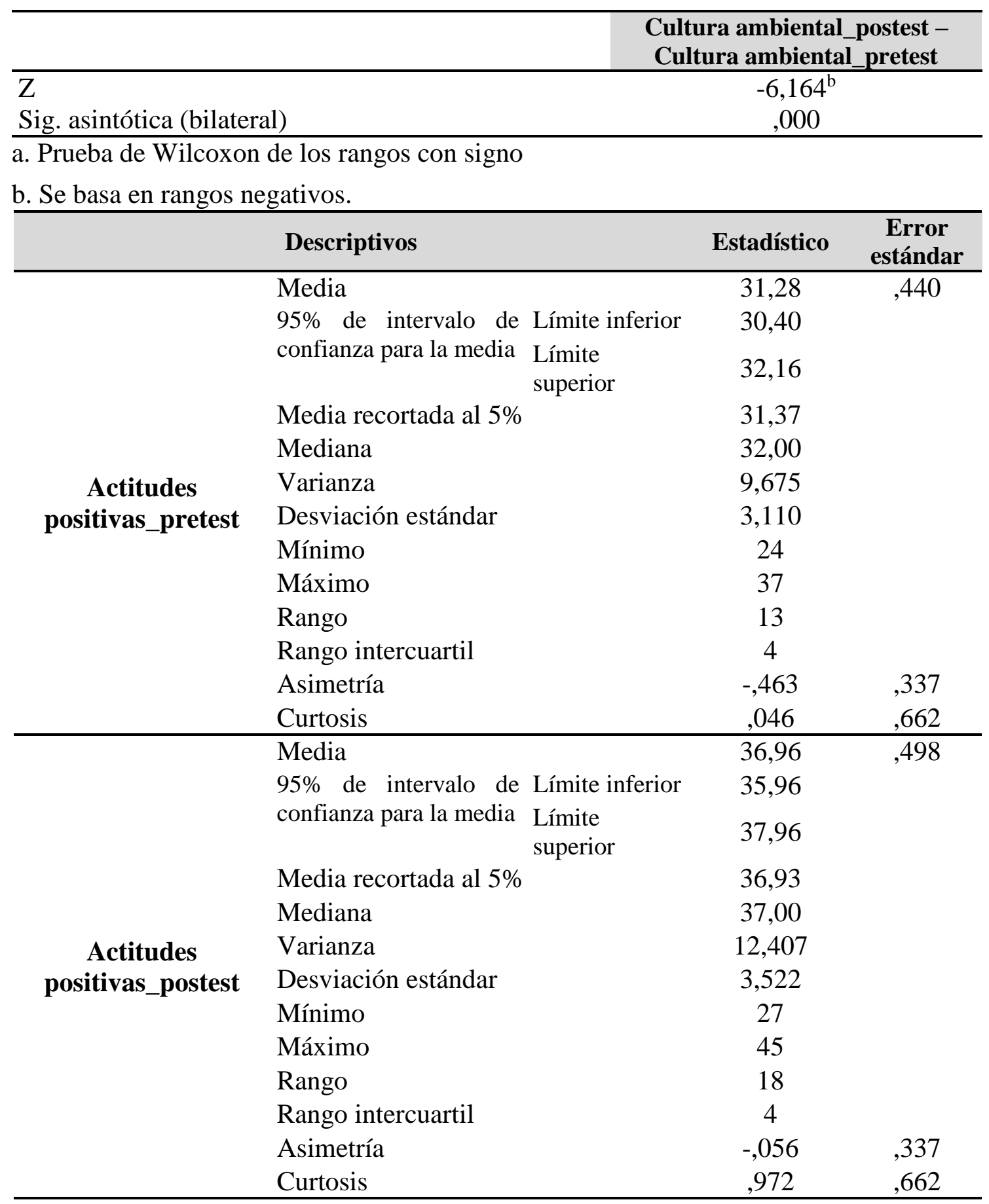


Finalmente, la variable cultura ambiental obtuvo tanto a nivel pretest y postest un 31.28 y 36.96 respectivamente en un rango especifico entre 9 y 45, lo que ha evidenciado una mejora significativa de 0.80 , así como la influencia del programa desarrollado en los estudiantes en la variable mencionada de una escuela básica regular.

\section{DISCUSIÓN}

En el trabajo realizado se ha verificado, de manera precisa, los objetivos planteados en la investigación, entorno a la relevancia del programa de recuperación de espacios libresáreas verdes y la cultura ambiental en los estudiantes de una escuela básica regular de la ciudad de Lima, estableciendo de manera pertinente la importancia entre dichas variables. Según los estudios que se han propuesto a continuación, sustentan que el uso de los programas de espacios libres de las áreas verdes se encuentra enlazado principalmente con la cultura ambiental. Por lo tanto, es necesario incluir dicho programa, en las sesiones de aprendizaje del Proyecto Educativo Institucional.

Al referirse a la dimensión actitudes positivas, ha coincido con la investigación desarrollada por Hernández y Reinoso (2018) en relación con la predisposición de los participantes voluntarios en las actividades vivenciales externas en perspectiva con el cuidado de los espacios públicos comunales. Del mismo modo, el estudio identificó que la motivación extrínseca es un factor fundamental para que se ejecuten acciones conjuntas en la que la mayoría son jóvenes y adolescentes. En ese sentido, los resultados han evidenciado que la influencia es notoria y que la presencia de las actividades externalizadas fomentará el acercamiento con el entorno y el conocimiento de la problemática ambiental para la búsqueda de soluciones efectivas.

Además, la dimensión intenciones de comportamiento se asocian con el estudio de Flores y Gutiérrez (2019) en la disposición de las actitudes de los participantes para poder ejecutar las acciones con mucho esmero y responsabilidad dentro de un plazo determinado y evitando situaciones de incomodidad entre los partícipes de la experiencia. Del mismo, modo, los resultados en esta dimensión han evidenciado que la presencia de los programas de recuperación no solo fortalecerá las competencias relacionadas con la cultura ambiental, sino que brindará una convivencia adecuada y respeto mutuo entre los estudiantes.

Asimismo, la dimensión conocimiento ambiental ha mostrado resultados interesantes entorno a la adquisición de las competencias y capacidades respectivas. En ese sentido, 
se ha coincidido con la propuesta investigativa de Moreno Fernández y Pérez García (2018) en que tanto docentes como directivos son los responsables en la planeación efectiva y pertinente de los contenidos y actitudes relacionadas con el medio ambiente, su conservación y la formulación de propuestas que conlleven a una cultura preventiva a corto y mediano plazo. En ese sentido, los resultados han mostrado una significancia adecuada y el desarrollo de programas de recuperación de espacios públicos implementan el conocimiento de los estudiantes ante la problemática ambiental actual.

Finalmente, la variable cultura ambiental, su mejora significativa en los resultados sea asocian al estudio desarrollado por Sabogal Dunin, Cuentas Romero, Tavera Medina y Vargas Chunga (2019) en concordancia con la implementacion de acciones y propuestas viables en la planificación curricular escolar, asi como el apoyo de instituciones como las Municipalidades para fomentar programas efectivos de prevencion, cuidado $\mathrm{y}$ concientizacion ciudadana del medio ambiente. Del mismo modo, se ha asociado con Dueñas (2015) quien ha sustentado la importancia que los docentes motiven a los estudiantes mediante la formulacion de actividades vivenciales externas. Por ello, los resultados evidenciaron que la cultura ambiental involucra participacion, conocimiento, actitudes, intenciones, comportamientos direccionados a la mejora del entorno en el que habita y se desenvuelve a diario.

\section{CONCLUSIONES}

El propósito de la investigación fue determinar la influencia de un programa educativo para fomentar la variable cultura ambiental en estudiantes de educación básica regular. De esta manera, el estudio evidencia la influencia del programa de recuperación de espacios libres de las áreas verdes en la variable mencionada.

Asimismo, la investigación ha mostrado que el fomento de la cultura ambiental mediante el programa formulado y desarrollado predispuso las actitudes positivas, el buen trato y respeto mutuo entre los estudiantes participantes. También, se ha podido evidenciar que las actividades que forman parte del programa han permitido el fortalecimiento del conocimiento ambiental y de temáticas afines como la cultura preventiva, normativas de protección y cuidado de los espacios libres que fueron desarrollados de forma optima por los estudiantes de educación básica regular.

Por otro lado, es relevante acotar que el abordaje del estudio de las intenciones del comportamiento de los participantes en programas como el desarrollado en la presente 
investigación ha despertado mucho interés en la actualidad, puesto que el desarrollo de la cultura ambiental debe partir desde la escuela y la familia, entornos fundamentales para el proceso formativo de las personas en toda sociedad.

\section{REFERENCIAS BIBLIOGRAFICAS}

Abasto, P. (2020). Cultura ambiental de estudiantes del nivel secundario. El caso de una escuela del conurbano bonaerense en Argentina. Revista estudios ambientales, 8(2), 45-61. https://doi.org/10.47069/estudios-ambientales.v8i2.824

Aguado, I., Barrutia, J., \& Echebarria, C. (2017). Anillos Verdes: algunas experiencias europeas. Boletín De La Asociación De Geografos españoles, (73), 33-60. https://doi.org/10.21138/bage.2408

Cabrera, O. H. (2021). La educación ambiental como base cultural y estrategia para mejorar actitudes ecológicas en estudiantes. Ciencia Latina Revista Científica Multidisciplinar, 5(4), 5559-5572. https://doi.org/10.37811/cl_rcm.v5i4.707

Capristan, R. (2017). Manejo de áreas verdes en el distrito de Chaclacayo. [Tesis de Pregrado. Universidad Nacional Agraria La Molina, Lima] http://repositorio.lamolina.edu.pe/handle/UNALM/3470

Checa, M. (2016). Las áreas verdes en la Ciudad de México. Las diversas escalas de una $\begin{array}{lllll}\text { geografía urbana. } & \text { Biblio } 34,798 .\end{array}$ http://revistes.ub.edu/index.php/b3w/article/view/26339/0

Dueñas, A. (2015). Gestión de áreas verdes y mejoramiento ambiental en docentes del distrito de San Juan de Miraflores - 2014. [Tesis de Maestría. Universidad Cesar Vallejo. Lima. Perú]. https://repositorio.ucv.edu.pe/handle/20.500.12692/5761

Equipo de la Federación Española de Municipios y Provincias. (2016). Guía de infraestructura verde municipal Dirección y redacción. http://www.redbiodiversidad.es/sites/default/files/GUIA_Biodiversidad_CAP ITULOS1_5.pdf

Farroñan, F. (2017). Valoración económica de escasas áreas verdes urbanas en el distrito de La Victoria, Chiclayo. [Tesis de pregrado. Universidad Católica Santo Toribio de Mogrovejo, Perú]. http://hdl.handle.net/20.500.12423/854

Flores, J., \& Gutiérrez, L. (2019). Factores que estimulan la actividad de innovación en América Latina: un enfoque VECM. Economía, sociedad y territorio, 19 (61), 373 - 403. http://dx.doi.org/10.22136/est20191366 
García, S., \& Guerrero, M. (2006). Indicadores de sustentabilidad ambiental en la gestión de espacios verdes. Revista de Geografía Norte Grande, 35 (1), 45 - 57. https://scielo.conicyt.cl/scielo.php?pid=S071834022006000100004\&script=sci_arttext

Guezel, R., \& Wickel, J. (2015). Riqueza ecológica marina y áreas vulnerables de la Bahía de Paracas. Saber y hacer, 2 (1), 50 - 64. https://revistas.usil.edu.pe/index.php/syh/article/view/41/40

Hernández, J., \& Reinoso, I., (2018). La educación ambiental y el trabajo comunitario. Estrategia desde el Centro Universitario Municipal. Revista Caribeña de Ciencias Sociales. 1-11. https://url2.cl/iPv51

Maciel, T., \& Barboza, B. (2015). Áreas verdes urbanas: Historia, conceptos e importancia ecológica. CES Revista, $29 \quad$ (1), 30-42. https://seer.cesjf.br/index.php/cesRevista/article/view/87/pdf_44

Menacho, J., \& Cadenillas, V. (2021). Viaje de estudios, organizadores gráficos en el pensamiento crítico en estudiantes universitarios. PsiqueMag,.10(1),76-89. https://doi.org/10.18050/psiquemag.v10i1.2641

Miranda, L. (2013). Cultura ambiental: un estudio desde las dimensiones de valor, creencias actitudes y comportamiento ambiental. Producción +Limpia, 8(2), 94 - 105. http://www.scielo.org.co/scielo.php?script=sci_arttext\&pid=S190904552013000200010\&lng=en\&tlng=es.

Moreno Fernández, O., \& Pérez García, F. (2018). Escuela y desarrollo comunitario: Educación ambiental ciudadanía en las aulas de secundaria. Revista mexicana de investigación educativa, $23 \quad$ (78), $\quad 905 \quad-935$. http://www.scielo.org.mx/pdf/rmie/v23n78/1405-6666-rmie-23-78-905.pdf

Núñez Aldaz, G., Hayk, P., \& Bejas Monzant, M. (2021). Enseñanza de la educación ambiental para el desarrollo sostenible en el Ecuador. Polo del Conocimiento, 59, 6, 821-832. http://dx.doi.org/10.23857/pc.v6i6.2789

Peña, V. (2015). Configuración espacial de las áreas verdes públicas en el ámbito distrital adyacente a la Costa Verde, Lima. [Tesis de pregrado. Universidad Nacional Agraria La Molina. Lima. Perú]. http://dx.doi.org/10.21704/ac.v76i1.764 
Quispe, E. (2017). Situación de las áreas verdes urbanas en Lima Metropolitana. [Tesis de pregrado. Universidad Nacional Agraria La Molina, Lima, Perú]. http://repositorio.lamolina.edu.pe/handle/UNALM/2990

Rojas, S. (2016). Relación de los flyers publicitarios y el aprendizaje de los visitantes del Parque Zonal Sinchi Roca en el cuidado de las áreas verdes en el distrito de Comas, Lima, 2016 [Tesis de pregrado. Universidad Cesar Vallejo, Perú]. https://repositorio.ucv.edu.pe/handle/20.500.12692/895

Sabogal Dunin, A., Cuentas Romero, M. A., Tavera Medina, T., \& Vargas Chunga, F. (2019). Espacios públicos: estudio del distrito de Santiago de Surco en Lima, Perú. Revista Kawsaypacha: Sociedad Y Medio Ambiente, (3), 105-138. https://doi.org/10.18800/kawsaypacha.201901.005

Sánchez Aizcorbe, V. (2017). ¿Qué tan saludables se encuentran nuestras áreas verdes?: estudio piloto usando diversidad de aves como indicador [Tesis de pregrado. Universidad Cayetano Heredia, Lima, Perú]. https://hdl.handle.net/20.500.12866/1326

Severiche, C., Gómez, E., \& Jaimes, J. (2016). La educación ambiental como base cultural y estrategia para el desarrollo sostenible. Telos, 18 (2), 266 - 281. https://www.redalyc.org/pdf/993/99345727007.pdf

Simoes, A., Yanes, L., \& Álvarez, D. (2019) Transversalidad de la educación ambiental para el desarrollo sostenible. Universidad y Sociedad, Cienfuegos, 11, 5, 2532. http://scielo.sld.cu/scielo.php?script=sci_arttext\&pid=S221836202019000500025

Vila, L. (2017). Implementación de manejo de residuos orgánicos en áreas verdes [Tesis de pregrado. Universidad Nacional Agraria La Molina, Lima, Perú]. http://repositorio.lamolina.edu.pe/handle/UNALM/3010 